\title{
We Asked the Experts: Toward Personalized Immunosuppression for Liver Transplant Recipients
}

\author{
Joey Lew ${ }^{1} \cdot$ Emily R. Perito $^{1} \cdot$ Sandy Feng ${ }^{1}$
}

Accepted: 3 December 2021/Published online: 17 January 2022

(C) The Author(s) under exclusive licence to Société Internationale de Chirurgie 2021

Optimal health after liver transplantation requires avoiding both graft and systemic complications, a goal that demands optimal management of immunosuppression. Immunosuppression regimens are similar across institutions: corticosteroids with or without antibody induction initially, tacrolimus for typically indefinite maintenance therapy, and a third agent such as mycophenolate mofetil for variable but often limited duration. Usually, the number and dosage of drugs are reduced over time, with an eye toward maintaining normal liver tests. Overall, this approach has worked well, yielding many additional years of both graft and patient life, with good quality of life.

However, excessive immunosuppression still risks declining renal function, recurrent infections, de novo malignancy, and other toxicities. On the other hand, inadequate immunosuppression risks allograft injury, rejection, and potential failure [1]. With suboptimal immunosuppression management being so consequential, it is nonideal that decision-making is usually guided by a few simple and crude parameters in transplant center protocols. These parameters, such as recipient age, liver disease etiology, time since transplantation, and liver test profiles, have suboptimal sensitivity and specificity for both efficacy (prevention of rejection) and safety (minimization of toxicity). Consequently, the application of protocolized regimens inevitably injures some recipients with too much immunosuppression and injures some allografts with too little.

The primary difficulty in optimizing immunosuppression is that we are unable to directly measure its impact.

Joey Lew

Joey.lew@ucsf.edu

1 School of Medicine, University of California, 505 Parnassus Ave, San Francisco, CA 94143, USA
Anti-hypertensives are dosed by checking blood pressure rather than awaiting strokes or syncope. We have no mechanism to assay the state of the immune system in a sufficiently sensitive or dynamic way. We instead await episodes of rejection, infections, or cancer. A secondary challenge is posed by the accuracy of rejection diagnosis. Suspicion is raised when liver tests rise; liver tests, however, may be abnormal for many reasons. Conversely, they may be completely normal when rejection is evident on biopsy, a situation termed "subclinical" rejection [2].

\section{Non-invasive biomarkers}

The shortcomings of liver tests to accurately reflect allograft health have motivated exploration of non-invasive biomarkers, including donor-specific antibody, donorderived cell-free DNA (dd-cfDNA), and liver stiffness measurements. Donor-specific antibody indicates a host immune system response specific for donor cells that could cause graft injury via antibody-mediated rejection. ddcfDNA, a circulating biomarker that has been extensively studied in kidney and heart transplant recipients, has recently been explored in liver transplant recipients. The over-arching hypothesis is that dd-cfDNA levels above baseline may indicate graft injury; they have been associated with elevated liver enzymes in the setting of acute rejection. Longitudinal trajectories of rising dd-cfDNA levels have been linked to graft injury, and a dd-cfDNA threshold has been suggested to differentiate allo-immune attack from other mechanisms of graft injury [3]. Imaging modalities including ultrasound elastography, acoustic radiation impulse frequency imaging, or magnetic resonance elastography can be used to measure liver stiffness, which varies with the degree of inflammation, edema, and/ 
or fibrosis. Intriguingly, an adult immunosuppression withdrawal trial recently showed that significantly increased stiffness corresponded with histological identification of not only rejection but also subclinical graft injury [1]. While each of these non-invasive metrics holds promise to reflect allograft health, additional rigorous, prospectively collected data are required to justify the use of biomarkers, individually or in combination, to guide decision-making regarding immunosuppression.

\section{Liver biopsy-lessons and limitations}

Given the lack of accurate non-invasive metrics, liver biopsy arguably remains the gold standard assessment of liver allograft health, and thus a critical guide when personalizing immunosuppression. However, even this "gold standard" has well-known shortcomings. First, there is a concern for sampling error, a risk potentially magnified by the patchy nature of some histopathology. Second, it is invasive; frequent use is impractical, even if reasonably safe. These factors contribute to immunosuppression decision-making inertia since, given normal liver tests, biopsy would be the only way to gauge the impact of any change. Third, and perhaps most importantly, the prognostic significance of subclinical histological abnormalities for future allograft outcomes remains uncertain. These caveats are, however, counterbalanced by a substantial literature on surveillance biopsies (biopsies done per center protocol in the setting of normal liver tests) performed in children with liver transplants. Multiple cross-sectional and single-center studies from Europe, Asia, and the USA have reported a high prevalence of both inflammation and fibrosis. The proportion of children with abnormalities increased with time after transplantation, raising concern that abnormalities might be progressive in nature.

One of our primary contributions has been to elucidate the etiology of the observed histological abnormalities by deeply interrogating a multi-center cohort of 157 biopsies collected from children at the time of normal liver tests. Hierarchical cluster analysis segregated the cohort into three clusters characterized by 1) interface activity with variable fibrosis; 2) fibrosis but without interface activity; and 3) essentially normal histology. Biopsies in cluster 1 uniquely had increased numbers of inflammatory cells and a transcriptional profile enriched in IFN- $\gamma$-regulated genes that were qualitatively identical to acute rejection, albeit attenuated [4]. The allo-immune nature of interface hepatitis has been confirmed in a parallel cohort of biopsies collected from adult liver transplant recipients [5]. The congruent results from these two studies beg the narrow question as to whether increasing immunosuppression might preserve allograft health and the broad question as to whether using surveillance biopsies to guide immunosuppression decision-making might yield benefit. The first report of utilizing surveillance findings to guide routine, standard of care, personalization of immunosuppression management for adult liver transplant recipients has recently reported favorable results [2]. Biopsy results led to changes for $79 \%$ of patients, with the dominant majority undergoing reduction. Reduction was associated with stabilized renal function, which differed significantly from the deteriorating renal function of a historical control cohort. Though not espousing a specific protocol, we feel that these promising results further suggest that liver biopsy remains a critical tool in guiding personalized immunosuppression regimens at this time.

Liver transplantation has resoundingly succeeded in saving lives-most patients and their grafts survive for many years. But many years is no longer sufficient; many decades are required. We can only achieve this with more sophisticated management of immunosuppression. We have already identified several tools that may better guide immunosuppression management than liver tests, but research must continue to not only fortify the toolbox but also teach us when and how, singly or in combination, to achieve the minimum immunosuppression necessary to maintain allograft integrity while optimizing patient well-being.

Funding This work did not receive grant or other funding support.

\section{Declarations}

Conflicts of interest The authors declare that they have no conflicts of interest.

\section{References}

1. Vionnet J, Miquel R, Abraldes JG et al (2021) Non-invasive alloimmune risk stratification of long-term liver transplant recipients. J Hepatol 75(6):1409-1419

2. Saunders EA, Engel B, Höfer A et al (2021) Outcome and safety of a surveillance biopsy guided personalized immunosuppression program after liver transplantation. Am J Transplant. https://doi. org/10.1111/ajt.16817

3. Levitsky J, Kandpal M, Guo K et al (2021) Donor-derived cell-free DNA levels predict graft injury in liver transplant recipients. Am J Transplant. https://doi.org/10.1111/ajt.16835

4. Feng S, Bucuvalas JC, Demetris AJ et al (2018) Evidence of chronic allograft injury in liver biopsies from long-term pediatric recipients of liver transplants. Gastroenterology 155:1838-1851

5. Londoño MC, Souza LN, Lozano JJ et al (2018) Molecular profiling of subclinical inflammatory lesions in long-term surviving adult liver transplant recipients. J Hepatol 69:626-634

Publisher's Note Springer Nature remains neutral with regard to jurisdictional claims in published maps and institutional affiliations. 